\title{
The Economic Cloud Servises and Data Inrtegrity for Uncertainity
}

\author{
Mahesh, M.Laxmaiah
}

\begin{abstract}
A broad research has prompted a general comprehension of vulnerability issues in various fields rang-ing from computational science to basic leadership in financial aspects. In any case, an investigation of vulnerability on enormous scale registering frameworks and distributed computing frameworks is restricted. The greater part of works inspect vulnerability wonders in clients' view of the characteristics, goals and activities of cloud suppliers. In this paper, we talk about the job of vulnerability in the asset and administration provisioning, protection, and so forth particularly, within the sight of the dangers of secrecy, trustworthiness, and accessibility. In this paper, we handle the examination question: what is the job of vulnerability in distributed computing administration and asset provisioning? We survey primary wellsprings of vulnerability, central methodologies for planning under vulnerability, for example, responsive, stochastic, fluffy, hearty, and so forth. We additionally talk about possibilities of these methodologies for booking distributed computing exercises under vulnerability, and address strategies for relieving work execution time vulnerability in the asset provisioning.
\end{abstract}

\section{INTRODUCTION}

THE quick Development of the Internet and the upward thrust of allocated computing have empowered a completely unique sample of acquiring and devouring Information Technology (IT) administrations. Distributed computing has carried new possibilities to the Information and Communication Technology (ICT) agency permitting companies to re-suitable their IT workplaces to cloud providers and steer clear of high priced impending ventures of installing place their very very own framework and resulting expenses of help and overhauls. By strategies for cloud administrations, cloud clients can get to all their required competencies (i.E., computational assets, facts, and packages ) over the Internet, use what they need, and pay for what they use without being worried about the hidden framework. Therefore, customers revel in the solace of general utilities, for example, water, energy, fuel, and communication. Focal factors, as an instance, an utility version however availability, versatility, and simplicity of the board have made an enterprise-big circulate within the direction of distributed computing preparations. As in line with a conjecture from International Data Corporation

Revised Version Manuscript Received on July 10, 2019.

Mahesh, Assistant Professor, CMR TECHNICAL CAMPUS, Kandla Koya, medchal, Hyderabad, Telangana, India (E-Mail kotha.mahesh528@gmail.com)

Dr.M.Laxmaiah, Professor \& Dean SAC CMR ENGINEERING COLLEGE Kandla Koya, medchal, Hyderabad, Telangana, India (E-mail: laxmanmettu.cse@gmail.com)
(IDC), the general spending on open cloud administrations is required to outperform $\$ 107$ billion out of 2017., Platform-asa-Service (PaaS), and Infrastructure-as-a-Service (IaaS). Among these severa types of conveying cloud administrations, IDC perceived the IaaS model as one of the fastest growing classifications with compound yearly improvement rate of $27.2 \%$. IaaS is a promising answer for empowering on-request get right of entry to to a flexible pool of configurable and virtual computational administrations (e.G., processing force, stockpiling, and systems) in a compensation as-you-move manner. An IaaS cloud specialist co-op possesses the statistics middle(s) and all of the required hardware and is in charge of facilitating, running, and looking after them.

Cloud innovations are generally utilized in the development of IT foundation of business, scholarly, government, and individuals as a legitimate answer for information stockpiling and preparing. While having numerous points of interest despite everything they have numerous disadvantages, particularly in the zones of security, dependability, execution of both processing and correspondence, to list only a couple. The change to enormous information and exa scale likewise represent various unavoidable logical and mechanical difficulties. In the distributed computing, administrations and assets are liable to extensive vulnerability during provisioning. Vulnerability might be introduced in various segments of the computational, correspondence, and putting away procedure. It requires deferring routine registering ideal models, adjusting current figuring models to this advancement, and planning novel asset the board methodologies to deal with vulnerability in a viable manner. The administration of cloud framework is a difficult undertaking. Unwavering quality, Safety, Quality of Service (QoS), execution stability, and value-effectiveness are sizable troubles in these frameworks. Accessible cloud models don't sufficient capture vulnerability, in homogeneity and dynamic execution modifications characteristic to non-uniform and shared foundations. To growth better comprehension of the con-groupings of a allotted computing vulnerability, we contemplate asset and management provisioning problems associated with existing cloud frameworks, as an example, half and 1/2 employer of open, personal and network ones.

There is an exploration on disbursed computing examining the vulnerability wonders in customers' impact of the traits, goals and activities of cloud providers, protection, safety, accessibility, and so on among extraordinary

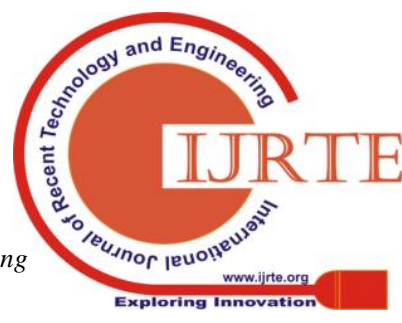


components of allotted computing (Trenz et al., 2013). Yet, the process of vulnerability in the asset and management provisioning, provider speculation and operational price, programming models, and so on have not yet been sufficient tended to inside the logical writing.

Outstanding burden in any such area can be modified considerably. It is hard to gauge runtime of occupations precisely, improve forecast with the aid of chronicled information, expectation revision, expectation fallback, and so on. (Ramírez et al., 2011). Flexibility has a higher repercussion on the character of administration, yet includes another factor of vulnerability. Vulnerability might be displayed in various parts of the computational and correspondence process. Significant inquiries are: the way specific unique calculation and correspondence qualities influence the productivity; how these attributes can be utilized to moderate vulnerability meeting wanted QoS requirements; how relating streamlining issue can be comprehended in proficient manner; how to convey versatile and powerful cloud conduct under vulnerabilities and explicit limitations, for example, spending plans, QoS, SLA, vitality costs; and so on. It is critical to ponder diverse stochastic, versatile, receptive, information free, and so forth asset provisioning calculations thinking about viable options in contrast to known deterministic advancement advances while keeping QoS.

\section{LITERATURE REVIEW}

Meinl et al. talk about the utilization of reservation frameworks in distributed computing conditions and call attention to the advantages for cloud merchants just as their clients. The creators dissected the utilization of subordinate evaluating procedures and yield the board to make a model propose a novel reservation instrument to shield the two suppliers and clients from the cost overhead of over-provisioning assets. In their booking component, shoppers can impart their remaining burden estimates as a prereservation and after that guarantee the pre-held assets if the need really emerges for the delicately saved assets in future. Pre-reservations catch the assessed measure of assets that will be required by a client at a given future purpose of time just as the likelihood of really requiring these assets. The proposed methodology incorporates instruments to abuse the expected data to be traded between the supplier and the client such that it use advantages of the two suppliers and clients.

So also, Lu et al. give an answer for the asset reservation issue in IaaS suppliers with constrained asset limit. Their proposed technique explores the attainability of each submitted reservation demand and if the supplier can't acknowledge the solicitation, an elective method for obliging the solicitation with in reverse Or ahead transferring in time is recommended. They use computational geometry to address the problem. Wang et al. Remember the asset reservation the executives problems inside cloud situations. They advise a flexible asset reservation technique by using specially tolerating reservation needs. The desire is made to that can be used in certifiable frameworks. Mohammadi et al.

augment the cloud dealer earnings while it guarantees the nature of management (QoS) for value-based applications.

Kantere et al. Exhibited an ideal comparing approach achieved thru the dynamic valuing for a booking administration presented by means of the cloud professional business enterprise. They advocate a unique cost request version supposed for a cloud shop and a dynamic estimating plan for inquiries accomplished within the cloud reserve. They likewise speak approximately subjective elements of the association that lets in the concept of patron loyalty together with cloud provider advantage amplification. A dynamic valuing plan appropriate for united cloud situations with sound and self-invested people wherein asset request and deliver range as customers be a part of and go away the framework has been proposed by way of Mihailescu and Teo. They analyze the presentation in their proposed system verification dynamic valuing model with fixed-fee usage primarily based comparing making use of reenactments, and exhibit that consumer welfare and the extent of fruitful solicitations are improved whilst their dynamic estimating model is attached. As the importance of dynamic valuing in cloud evaluating has been perceived by means of the writing, we commit the subsequent vicinity to speaking about and examining dynamic estimating related paintings..

Zheng et al. Audit the best in magnificence and diagrams an exploration guide on cloud administration association. They outline three studies troubles, i.E., QoS estimation, QoS alternate, and QoS requirement, for cloud administrations. Their examination indicates whilst one has no mastering of which methodology the adversary will play, a mixed system can accomplish a better utility than the concession method. Child et al. Propose a multi-difficulty exchange system to achieve a concession to the cost of an administration and when to utilize the administration. In their system an arrangement operator can simultaneously make various proposition in every exchange round. They likewise describe an utility capacity that models the inclinations of the client for various vacancies. A contextual investigation showing the advantages of utilizing their instrument with three estimating models of Amazon EC2 has been done.

Kliazovich et alpropose new Communication-Aware Directed Acyclic Graph (CA-DAG) model of cloud computing applications by presenting correspondence mindfulness, which over comes inadequacies of existing methodologies and permits to mit-igate vulnerability in increasingly proficient manner. It depends on a Directed Acyclic Graph, which alongside processing vertices has sepa-rate vertices to speak to interchanges. Such a representationallows settling on discrete asset distribution choices, assigning processors to deal with processing occupations and system assets for information transmissions. The proposed correspondence awaremodel makes space for enhancement of many existing arrangements to resource portion just as growing totally new schedul-ing plans of improved proficiency. 


\section{FEDERATED CLOUD ENVIRONMENTS}

Late examinations demonstrate the advantages of interconnecting cloud conditions and gift endeavors for the acknowledgment of mixed cloud condition. The blessings of interconnected cloud conditions for each cloud providers and their clients are diverse and there are primary inspirations for cloud interoperability if you want to in the end cause the Inter-Cloud. Before speakme about blessings of cloud interoperability, we inspect exclusive plausible cloud interoperability conditions.

\subsection{Motivations for Cloud Interoperability}

In this section, key advantages of cloud interoperability which gives basic inspirations to interconnected cloud conditions have been abridged (Figure 1.1 )..

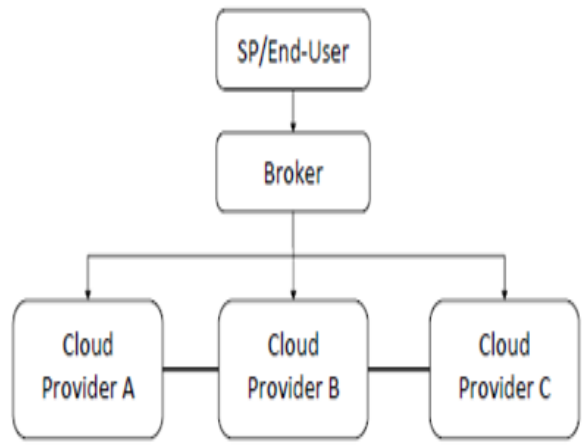

Fig. 1. Aggregated service broker scenario.

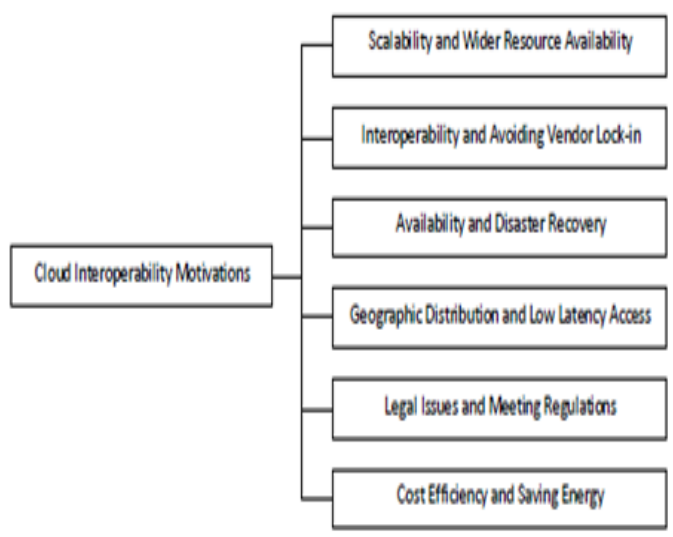

Figure 1.1: Cloud Interoperability Motivations.

\section{Scalability andWider Resource Availability}

Despite the fact that one of the key highlights of distributed computing is the deception of endless assets, limit in cloud supplier's server farms is restricted and in the long run can be completely used. Development in the size of existing applications or flood popular for an administration may bring about prompt need of extra limit in the server farm. Current specialist co-ops handle this issue by over-provisioning of server farm limit. That is, the normal interest of the framework is a few times littler than the limit of their registering foundation. This procedure and the expense of its activity establish an enormous cost for cloud proprietors. Real use examples of some certifiable application administrations differ with time and more often than not in erratic ways. Consequently, sudden burdens can possibly overburden a solitary cloud supplier and lead to problematic and interfered with administrations.

It is excessively prohibitive as far as little size or private mists. On the off chance that cloud suppliers had the option to powerfully scale up or down their server farm limit, they could set aside significant measure of cash and conquer the above issue. Adaptable provisioning of use benefits under factor outstanding task at hand, asset, and system conditions is encouraged by interoperation of the mists. Cloud Federation enables the crest to load dealing with limit of each endeavor cloud by asset sharing, without wanting to keep up or direct any extra processing hubs or servers.

\section{UNCERTAINITY}

Regardless of broad research of vulnerability issues in various fields in the previous decades going from material science, computational science to basic leadership in financial aspects and sociologies, an investigation of vulnerability for distributed computing frameworks is as yet not accessible. There are various kinds of vulnerabilities related with distributed computing, and one should represent parts of vulnerability in evaluating the effective administration provisioning. Moderating effect of vulnerability on the presentation, dependability, security, and strength of cloud frameworks is quickly developing examination point. Vulnerability examination ought to turn into a fundamental piece of structure of asset and administration provisioning systems. This paper displays our comprehension of how to demonstrate distributed computing with vulnerability tending to asset provisioning in half and half private-open cloud condition, dynamic self-versatile dispersed facilitating, flexible mists, and streamlining of related issues to convey hearty asset the executives arrangements, where the fundamental target isn't to locate an outright ideal but instead arrangements that carry on great and obtuse toward various dubious ties. Superior targets could prompts too unsafe execution arrangements.

\subsection{Uncertainty in long-term predictions}

Over-burden or underload recognition dependent on long haul forecasts conveys with it the vulnerability of right expectations, which can prompt mistaken choices. To consider the vulnerability of long haul forecasts, the above location components are enlarged with the consideration of a probabilistic conveyance model of the expectation mistake.

To start with, the likelihood thickness capacity of the expectation mistake for each forecast time interim is assessed. Since the likelihood conveyance of the expectation blunder isn't known ahead of time and various remaining burdens can have various disseminations, a non-parametric technique to fabricate the thickness work online is required. In this work, a non-parametric technique for likelihood thickness work estimation dependent on portion thickness estimation is utilized. It gauges the likelihood thickness capacity of the forecast mistake each time interim dependent on a background marked by past expectation blunders. In this work, the likelihood thickness capacity of the

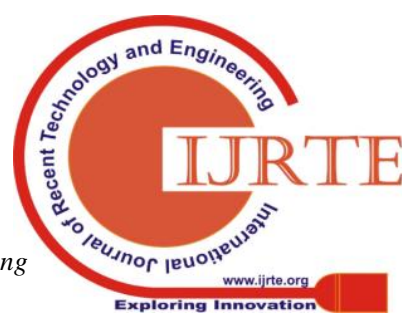


supreme estimation of the forecast blunder is utilized.

\subsection{Probabilistic overload detection}

In light of the likelihood thickness capacity of the forecast blunder, it tends to be assessed probabilistically, for each anticipated time interim, if the future complete CPU use will be more noteworthy than the over-burden edge. In the accompanying, for accommodation, the future all out CPU utilization is simply called the future CPU use. This is accomplished by Algorithm 1 that profits genuine or false with some likelihood whether the future CPU utilization will be more noteworthy than the over-burden limit.

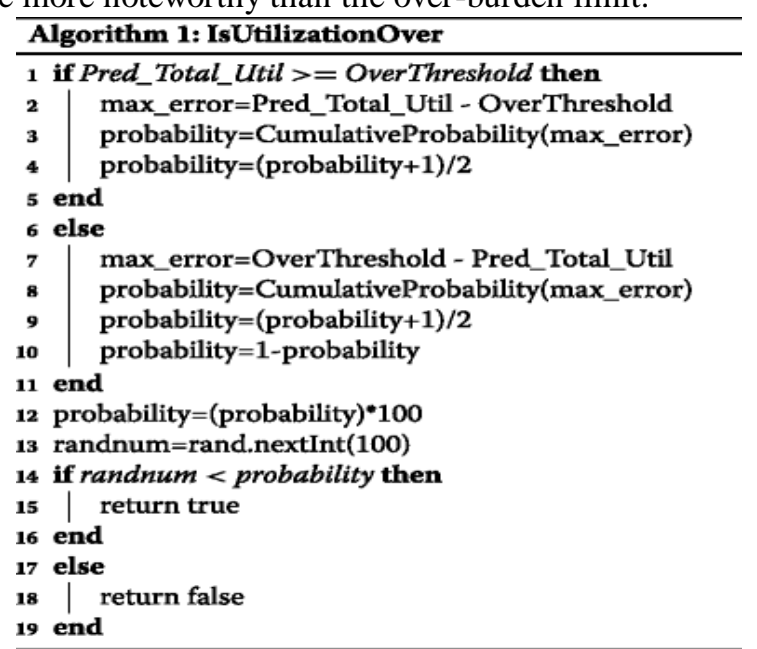

To begin with, the calculation finds the likelihood that the future CPU utilization will be more noteworthy than the over-burden edge. On the off chance that the anticipated CPU use is more prominent than the over-burden edge, the distinction, called max_error, between the anticipated CPU use and over-burden limit, is found. For the future CPU use to be more noteworthy than the over-burden edge, the outright estimation of the blunder (i.e., the distinction among anticipated and future worth) ought to be not exactly max_error. In light of a combined conveyance capacity of the forecast blunder, the likelihood that the expectation mistake is not exactly max_error, i.e., the future CPU utilization is more prominent than the over-burden limit, is found. Since it can happen that the future CPU use will be more noteworthy than the over-burden limit, and furthermore that the expectation mistake will be more noteworthy than max_error, the likelihood this occurs, given as (1-likelihood)/2, is added to the determined likelihood to yield the last likelihood (probability+1)/2. In the event that the anticipated CPU use is not exactly the over-burden limit, by a similar methodology, first, the likelihood that the future CPU utilization will be not exactly the over-burden edge is found. At that point, the likelihood that the future CPU use will be more prominent than the over-burden limit is given as (1-likelihood). At last, the calculation returns valid with the assessed likelihood.

\subsection{Reliability and privacy under uncertainty}

So as to build unwavering quality and classification of the dataprocessing and putting away, six fundamental methodologies are connected: information repli-cation, mystery sharing plans, repetitive buildup number system,erasure code, recovering code, and homomorphic encryption.

\subsection{Data replication}

The precept focal factors are excessive unwavering splendid and plausibility to ace cess the records in disseminated layout. The negative aspects are dramatic increase in information volumes and want to ensure each replication (Ghe-mawat et al.); (Abu-Libdeh et al.).

\subsection{Secret sharing schemes (SSS)}

SSS, for example, Shamir, Blackly, and so forth are techniques with the useful resource of which a issuer distributes gives to beneficiaries with the quit cause that lone approved subsets of beneficiaries can reproduce the thriller. They are enormous devices in cryptography and applied in plenty of comfortable conventions, e.G., famous expert device for multiparty calculation, Byzantine expertise, threshold cryptography, get to control, belongings based totally certainly encryption, and gen-eralized careless alternate. They permit to accumulate comfy allotted storage frameworks (Gomathisankaran et al.). Notwithstanding, if SSS is not homomorphic, calculation over encoded records isn't always possible or complicated. For the development of a completely homomorphic encryption, (Asmuth et al.) and (Mignotte, M.) suggest plans relying on RNS. The RNS allows to convey together a completely homomorphic ciphers due to the houses of the parallelism of math operations, which enhance execution and building up a homomorphic encryption this is asymptotically first-rate and modified, contingent upon the undertaking. Redundant Residue Number System (RRNS). In this framework, precise variety is spoken to as deposits concerning a moduli set. Thus the number may be element in-to a few littler numbers which may be loose and duties may be practiced on them one after the other and concurrently which makes the calculations much less hard and masses quicker. Repetition of buildups permits to gather dependable information getting ready framework with one-of-a-type blunder region and correction (Chessa et al.), (Celesti et al.). As indicated by way of RRNS property, at the off hazard that the amount of manage modules is $r$, at that point the tool can end up aware of $r$ and right $r-1$ mistakes. For mistake detachment and correction, projection strategies are utilized, wherein the quantity of calculated projections becomes exponentially depending upon the estimation of $\mathrm{r}$. As a result RRNS is unfeasible with out critical streamlining. 


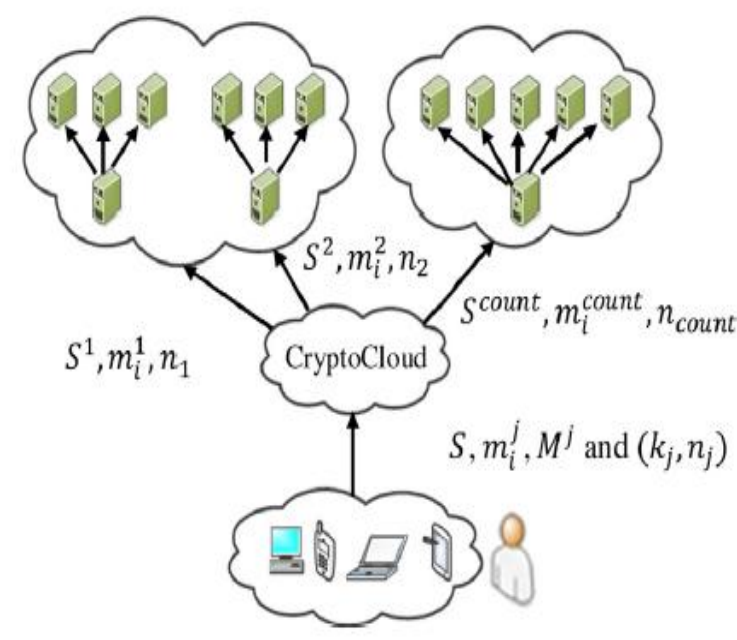

Fig. 2. Multilevel data encryption.

\subsection{Programming Uncertainty}

Vulnerability comprehension wishes to prompt disclosures in a way to configuration cloud programs in effective way. The majority of cloud applications require accessibility of correspondence assets for facts trade among assignments, with databases or stop customers. In any case, suppliers possibly may not recognise the quantity of information that may be overseen, or quantity of calculation required via assignments. For example, each time whilst a patron requires a status of a ledger, it is able to set apart brilliant attempt for its conveyance. Just couple of methodologies thinks about correspondence stipulations and frequently in a profoundly theoretical way. In addition, if applications are to apply the Cloud, their execution circumstance isn't always recognised at development time - the quantity of available machines, their place, their capacities, the machine topology, and powerful correspondence transfer speed can't be recognised ahead. When all is said in done, an execution domain contrasts for every application/management conjuring. To control this elements, both developers have to expressly compose versatile projects or cloud programming need to arrangement with the vulnerability.

The customer versatile arrangements depend on wonderful programming exertion. For a powerful usage of the Cloud, the tasks should be decoupled from the execution situation. Projects must be created for uniform and unsurprising virtual administrations, on this way, enhancing their advancement. Cloud application model desires to permit extraordinary nation portrayal of registering and correspondence dependent on the idea of the issue, and free of the executing circumstance. Mapping calculation on machines, adjusting the lots amongst diverse machines, expelling inaccessible machines from a calculation, mapping correspondence undertakings and adjusting the correspondence loads among various connections have straightforwardly be given by way of the runtime framework.

\subsection{The Sources of Uncertainty Perceptions}

In accordance with the workplace rationale, we advocate that vulnerability observations are confirmed in customer's worries that the administration pleasant (for instance safety, protection, accessibility) is not met by the supplier both due to hid facts (for instance the provider does no longer have the characteristics) or shrouded activities (as an example dealer acts craftily). As overviews on internet commercial enterprise choice discover, the maximum considerable explanations in the back of staying faraway from on-line exchanges are not beneficial but as an alternative are diagnosed with clients' lack of command over their very own information (Hoffman et al., 1999). Data safety and statistics safety worries are proven to be two noteworthy resources why customers are dubious about on line exchanges (Pavlou et al. 2007). From a head expert factor of view, we characterize records safety issues because the patron's conviction about a cloud supplier's failure (opposed desire) and reluctance (moral peril) to shield man or woman statistics from sick-advised use, revelation to outsiders, and optionally available use without the patron's assent (Pavlou et al., 2007). These safety worries are relied upon to a exceptional quantity upload to the customers' vulnerability recognitions within the cloud supplier purchaser dating:

H1: Users' degree of fulfillment with disbursed storage management is decidedly linked with their goal to keep making use of the administration.

H2: Users' apparent vulnerability of making use of the dispensed garage management is adversely related with their degree of achievement.

H3a: Information protection concerns decidedly effect a patron's apparent vulnerability. Data security concerns are characterised because the consumer's conviction about the cloud supplier's powerlessness and reluctance to defend client's close to domestic data from protection breaks in the course of transmission and capability (Salisbury et al., 2001). Because of the data asymmetry, the purchaser can scarcely skip judgment on whether or not safety ruptures take place (negative choice) and whether the right aversion measures are set up (ethical danger). The associated protection concerns add to the obvious vulnerability of the client:

H3b: Information security issues decidedly effect a consumer's apparent vulnerability. Other than the modern-day builds of facts protection worries and data safety concerns, vulnerability in dispensed computing provider consumer connections is proposed to likewise emerge from issues on the accessibility simply because the versatility of the management. Truth be advised, for you to rely upon a distributed garage supplier, customers assume a stable uptime of the distributed storage management (Bitcurrent, 2011). Subsequently, accessibility issues are of accelerated significance for this sort of management. Conversely, dispensed garage clients are less concerned whether stockpiling may be flexibly provisioned and discharged or now not in view that the degree of capacity they can utilize is frequently restrained by using the measure of ability on their community PC. Subsequently, we did exclude adaptability 


\section{The Economic Cloud Servises And Data Inrtegrity For Uncertainity}

issues in our exam. Predictable with the meanings of safety and security issues, we symbolize accessibility worries because the client's conviction approximately the powerlessness and reluctance of a supplier to deliver full management accessibility. The information asymmetry using the accessibility worries depend upon the restricted potential outcomes to evaluate uptime (unfriendly dedication) and the trouble to survey the unwavering nice and gave help limits (ethical chance). As needs be, we advocate that rising accessibility worries increment the purchaser' saw vulnerability:

H3c: Availability issues decidedly effect a consumer's obvious vulnerability.

\subsection{Mitigators of Perceived Uncertainty}

As there's no perfect correspondence between actual flagging speculations and motivation structures and their Sirdeshmukh, 2000), this exam method to test some consumer associated factors that effect clients' abstract evaluation of sign and motivators in place of considering the adequacy of express signal or impetus structures (Bergen et al., 1992).

Predictable to past studies (Pavlou et al., 2007; Singh and Sirdeshmukh, 2000), we advocate 3 userrelated factors consider, peer appropriation and exchanging expenses which are prevalent to effect client's worries on records protection, statistics security and accessibility.

Trust is a vast perspective in situations that encompass 2008). We represent trust as a number of specific convictions inside the dispensed garage supplier, to be unique fitness (the distributed storage dealer can address the purchaser's issues), altruism (the dealer minds and is roused to behave to the client's gain), and honesty (the supplier is simple and remains trustworthy to its commitments) (Gefen et al., 2003). Steady to past examinations (Pavlou et al., 2007), we endorse that accept as true with affects how customer's get each flagging and motivation systems and therefore, mitigates every one of the three proposed wellsprings of noticed vulnerability. believe to determine their trouble with hid information. Ordinarily, providers signal their kindheartedness, talent and trustworthiness through demonstrating their procedural decency concerning security coverage (as an example via dispensing an information safety proclamation), safety insurance (for instance thru having outsiders confirm their safety approach) and dependability (for instance through distributing beyond down activities) (Pavlou et al., 2007). Then again, believe is proposed to decrease fears of hid sports. Clients are satisfied to discover their data to the cloud supplier if the provider is seemed to be skillful, to be of uprightness, and to be considerate. Understanding that reliable, highquality suppliers might not endanger their notoriety, clients will be less concerned to utilize the suppliers send signal and motivations, customers will simply emotional evaluation by way of customers (Singh and reliance on an outsider (Gefen et al., 2003; Gefen et al.,

From one perspective, clients are proposed to rely upon distributed storage management. In entirety, whilst all

have faith in those originating from providers they believe (Pavlou et al., 2007). Following our line of argumentation, we suggest:

H4a: Trust adversely affects a purchaser's records protection worries. H4b: Trust adversely impacts a customer's facts protection worries.

H4c: Trust adversely affects a patron's accessibility concerns. Earlier studies on IT use behavior recommends that underneath states of vulnerability person's convictions, for example, concerns approximately utilising allotted garage administrations are impacted by the diploma to which a massive interpersonal employer, for instance, associates or partners has obtained the management (Karahanna et al., 1999), characterized as friend selection (Zhu et al., 2006). Along those traces, we draw upon crafted with the aid of Fulk (1993) who has proven the social effect wonder in placing of an exam on e-mail use internal an affiliation. In light of her paintings, we advise that customers are sure to decidedly bypass judgment on signal and motivations if their companions are utilising a similar distributed garage provider because of the increasing ramifications for the dealer whilst hid information and activities are disclosed. Subsequently, the greater friends make use of the disbursed storage provider, the much less concerned are people to make use of the administration.

H5b: Peer appropriation adversely impacts a consumer's facts security concerns.

H5c: Peer appropriation adversely impacts a customer's accessibility concerns.

A lock-in situation speaks to defenselessness since the purchaser cannot go away the cloud dealer client dating with out acquiring economic misfortunes (Ray et al., 2012). The unique concept of the powerlessness has huge ramifications for the way customers can reply on hid activities of dispensed garage providers (Wathne and Heide, 2000). Two styles of exchanging prices can for the maximum part be separated: sunk charges and procedural replacing charges (Beatty et al. 2012; Jones et al., 2007). Because of the low task important to make use of a allotted garage management, procedural replacing expenses are maximum significant in this case. Procedural replacing charges encompass the time, exertion, and hassle of finding and adjusting to every other dealer (Jones et al., 2007). For distributed storage administrations, embracing infers two noteworthy advances: convalescing the data from the allotted garage provider and moving it to the brand new administration. Exchanging costs for disbursed garage administrations can besides be of social nature (Jones et al., 2007), as an instance meditated inside the lost gain of supplying files to special customers of a similar management. These replacing fees may be adjusted via the dispensed storage expert employer thru diverse terms of management, receptiveness of the interfaces, and many others. 


\section{METHODOLOGY}

The speculations inferred in the past vicinity were tried close to distributed garage administrations making use of take a look at facts from an online poll among genuine clients. In the accompanying, we depict our estimation development just as the observe sending and facts accumulation techniques. All estimates applied in our investigation had been acquired from current measures. The measures and their resources are appeared inside the Appendix. In any case, they had been adjusted to the putting of our examination. Along these traces, we related a progression of device to guarantee the precise estimation of our builds. Above all else, definition, space and dimensionality of the develops were examined and characterized. We furthermore guaranteed construct legitimacy and fathomability using four raters and an open arranging approach (Moore and Benbasat, 1991). We pursued the methodology by means of MacKenzie et al. (2011) to approve the substance legitimacy of our develops by means of having the cover among the matters and the construct area evaluated by a second arrangement of raters. The starter device turned into then pilot tried with 25 participants. After the pretest, the respondents had been approached to present open complaint with admire to association of the study, by and large time, and specific troubles they encountered. Following the pretest, the tool become abbreviated, refined, and accredited for its actual residences.

The final study became directed online among March and April 2012. The on line look at was very suitable to deal with customers of disbursed storage administrations because the same old on-line get entry to is an critical to be used of such an administration. On the number one page of the assessment, the that means of disbursed storage management become given and participants had been requested which dispensed storage administration they utilize maximum (assuming any). All inquiries have been then certainly adjusted to allude to their particular management. The connection to the overview changed into conveyed to 527 Bachelor, Master and $\mathrm{PhD}$ understudies at a first-rate enterprise university in Germany. The opportunity to win Amazon vouchers changed into set as motivating force for guide. On the entire, 163 completed reactions were accrued. The season of consummation was recorded to take out reactions with curiously low fruition times. Besides, participants that did now not make use of any distributed garage administration have been evacuated. At closing, 143 reactions have been considered usable, prompting a $27.1 \%$ compelling response charge. Respondents were $37 \%$ female (63\% male) between the a while of 21 and 34 years (Mean: 26; Median: 26). Since the socioeconomics of the non-respondents had been difficult to understand, we analyzed past due respondents' socioeconomics, who regularly are like non-respondents (Miller and Smith, 1983), to the ones that spoke back inside the start of the duration.

\section{CLOUD STORAGE ISSUES AND NEED OF DATA INTEGRITY SCHEMES \& RESULTS}

Distributed storage is beneficial for the two clients and expert co-ops. Cloud clients result in least capital hypothesis and are assuaged from basis protection responsibility. By using allotted garage, a customer is given location self reliant get entry to to administrations (Armbrust et al., 2009). In the period in-between, a CSP increases high profits over on hand framework by way of sharing it among severa clients. This peculiar state of asset usage prompts advanced vitality usage. Notwithstanding those advantages, disbursed storage has a few natural shortcomings, as CSP is constantly time-honored untrusting, which can also have malevolent inspirations. A CSP may also keep on malevolently either purposefully (charging for all records and erasing the unaccessed information after use measurements exam or maintaining the less reproductions consistent with obligation) or accidentally (expert agency himself is ignorant if awful segments made in circle or difficult plate crashes).The client's issues about disbursed storage are given underneath:

1. Unauthorized physical get right of entry to

In cloud-based capacity, statistics might be dispersed over various servers and locations. The patron loses command over his information and cannot look into the records connects outwardly. Consequently, the danger of unapproved physical get admission to is one of the problems of distributed storage. Re-designation of more room, switch of vintage tools or reuse of drives can result in information ruptures.

2. Cloud garage company espionage

Clients are normally concerned that professional businesses can also undertaking to accumulate touchy records from their statistics without his assent or studying Particularly when statistics consists of non-public records, for example, Visa numbers, therapeutic statistics, and so on., customers falter to embrace distributed storage.

Three. Data lock-in

No gauges exist that represent how records have to be placed away and saved up inside the cloud. Each CSP has its restrictive layout for statistics stockpiling. In this manner, clients get sure to a selected CSP and changing to every other isn't always honest. Successive changes in cloud-based totally administrations likewise cause problems for clients.

4. Cloud garage issuer balance

Distributed garage professes to present excessive accessibility. Be that as it is able to, shouldn't some thing be stated about the CSP soundness? This carries:

- how many administrations given by a CSP are steady and how frequently do they alternate?

- Is the CSP equipped for moderating catastrophic occasions?

- Reliability of other organisation's administrations procured by means of the CSP?

- What if the CSP fails?

Every this type of inquiries can result in consumer dithering for the reception of disbursed garage. 


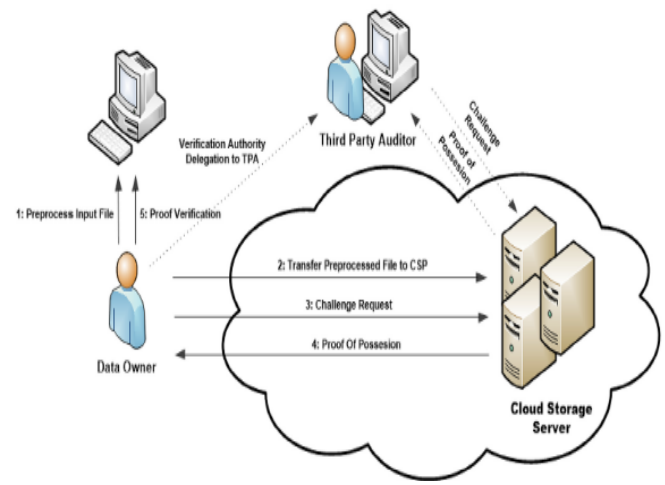

Fig. 3 - Data integrity scheme protocol.

There are two phases in a data integrity protocol:

1. Preprocessing stage: information are preprocessed, and some extra metadata are created. At that point, both the information and metadata are redistributed to the CSP.

2. Confirmation stage: an evaluator sends a test solicitation to the CSP who creates the verification of ownership utilizing the information and metadata and gives it to the reviewer. At that point, the examiner confirms the confirmation, to guarantee that the information uprightness is unblemished. With the assistance of information respectability plots, any information defilement or erasure can be convenient distinguished and in this way essential measures can be taken to recoup the data.To build up a superior comprehension of information uprightness plans, we have characterized a topical scientific classification dependent on the idea of information they bargain, the nature of metadata they use, their capacities, execution natives utilized and sending situations..

\subsection{Taxonomy of data integrity schemes}

Coming up next are the traits mulled over for characterizing the scientific classification of information respectability plans. These qualities depict the capacities of any plan as well as give a thought regarding their impediments in handy situations. Fig. 4 demonstrates the abridged perspective on plans with reference to these characteristics.

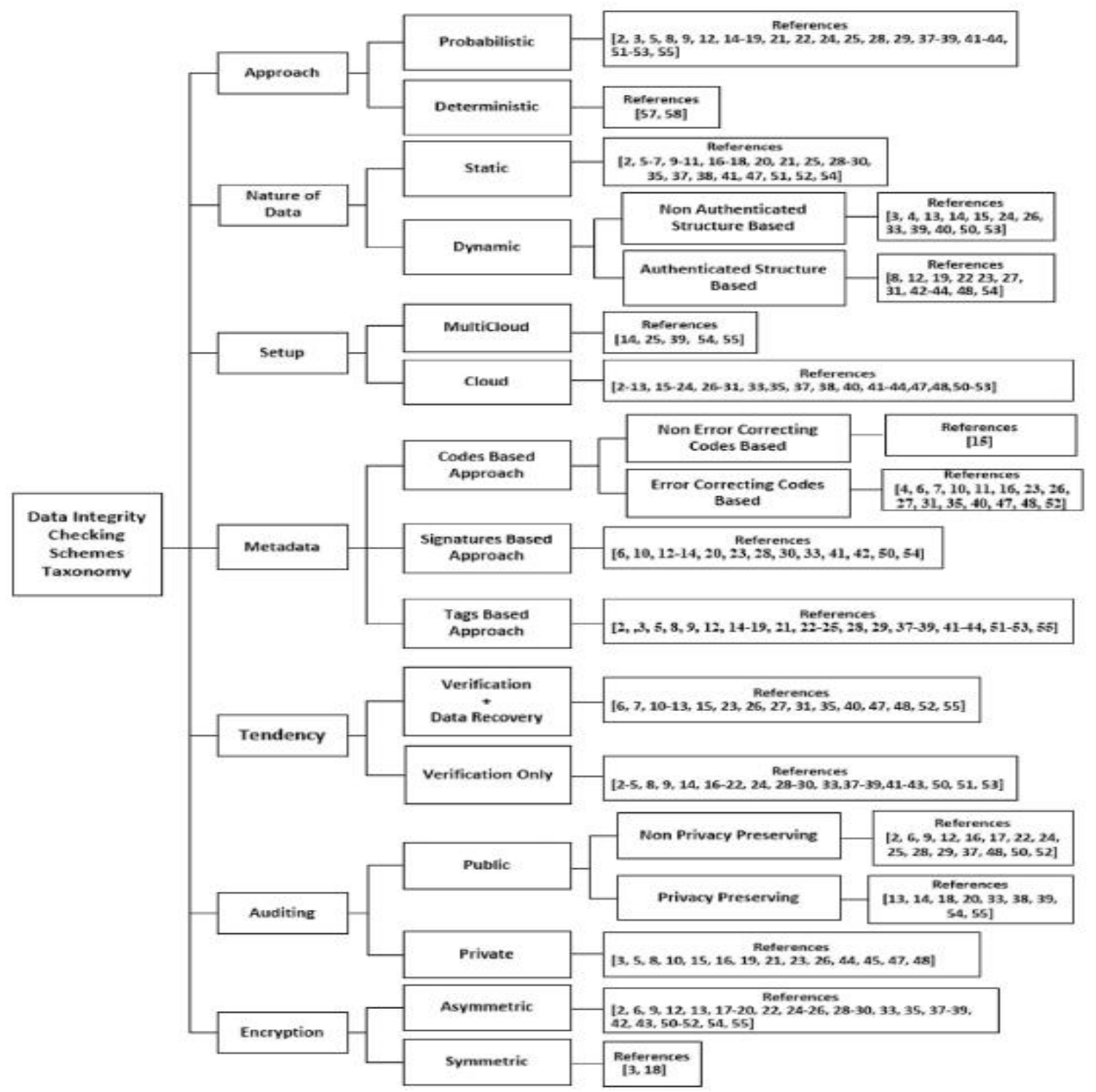

Fig. 4 - Taxonomy of data integrity schemes. 

- Approach: The method alludes to the concept of certification given by way of an facts uprightness plot, which can be deterministic or probabilistic. Deterministic plans (Deswarte et al., 2004; Han et al., 2014) need to get to the full report to decide the uprightness and supply one hundred\% belonging ensure. Though, probabilistic plans (Ateniese et al., 2007, 2008) make use of haphazardly picked squares of data to test the trustworthiness and deliver beneath $100 \%$ assurance. Deterministic plans are not appropriate for huge size data, as an example files having records size in GBs or TBs as respectability take a look at of this type of record takes an extra of time. This can spark off a constraint on how often the purchaser can perform uprightness check. Along those strains, probabilistic plans are steadily proper for large documents. Then again, deterministic affirmation is suitable megabytes. Computerized libraries (virtual books, articles, photographs, and so on.) basically have little size facts but are good sized in wide variety. For such form of data, guarantees. Ateniese et al. (2007) proposed the foremost probabilistic trustworthiness conspire in 2007, which utilized arbitrary inspecting of squares instead of perusing the whole document. By reviewing four.6\% of all out squares of a document, it gives ninety nine $\%$ belonging make certain. This plan gave the base to probabilistic honesty checking plans, yet its presentation diminishes as the size of facts increment.

- Setup: The association alludes to the concept of situation in which information respectability plan may be despatched. Since records are placed over the cloud, which can be simple

(impartial, giving a extensive range of skills itself) or "cross breed/haze of cloud/multi-cloud" (coping with positive belongings internal and other are given remotely; Zhu et al., 2011), nature of arrangement prompts some more stipulations in information respectability plans. For example, a cloud arrangement can bolster replication inner a server and crosswise over servers, expanding the respectability checking from unmarried to numerous copies of data. Curtmola et al's. (2008) proposed plan is assessed "severa reproductions provable records ownership conspire (MR-PDP)", that's geared up for producing new imitations on hobby if there have to arise an occurrence of any debasement or cancellation. Utilization of MR-PDP is limited to a solitary cloud havingmultiple capability servers but isn't extendable to a multi-cloud situation. Nooks et al. (2009a) concentrated on excessive accessibility by abusing the extra inner a server and crosswise over servers. Peterson et al. (2011) accelerated information uprightness and blanketed help for take a look at of SLA to guarantee that redistributed statistics are in indicated topographical limit. Zhu et al. (2012) provided features of the excessive for littler measured files having the scale in couple of check out papers) and media substance (melodies, deterministic plans are best to give a hundred\% belonging

protection, advanced, and simple affirmation for PDP model close to multi-cloud.

\section{CONCLUSIONS}

The vulnerability is a significant issue that influences computing efficiency carrying extra difficulties to planning problems. It requires structuring novel asset the board techniques to handle vulnerability in a successful manner. We deal with regions such as resource provisioning, application execution, and communication provisioning. They need to present the capability to dynamically allocate, oversee property in mild of changing interest pat-terns continuously, and gradually regulate them to adapt with unique terrific burdens and cloud residences to assure QoS. We audit and characterize distributed computing vulnerability, and examine ways to deal with its moderation. We feature developing patterns, future bearings in this field, job of vulnerability from suppliers, client and expediting viewpoints; dynamic asset and administration provisioning procedures; and programming, in nearness of vulnerability.

\section{REFERENCES}

1.M. Trenz, J.C. Huntgeburth, D. Veit, The role of uncertainty In cloud computingcontinuance: antecedents, mitigators, and consequences, Proceedings of the21st European Conference on Information Systems (2013) 147.

2.Tchernykh, U. Schwiegelsohn, V. Alexandrov, E.-G. Talbi, Towardsunderstanding uncertainty in cloud computing resource provisioning, Proc.Comput. Sci. 51 (2015) 1772-1781.

3.J. Ramirez, A. Tchernykh, R. Yahyapour, U. Schwiegelshohn, A. Quezada, J.Gonzalez, A. Hirales, Job allocation strategies with user run time estimates foronline scheduling in hierarchical grids, J. Grid Comput. 9 (2011) 95-116.

4.A. Tychinsky, Innovation Management of Companies: Modern Approaches,Algorithms, Experience, 2006 (Online]. Available: http://www.aup.ru/books/m87/ [accessed 29 June 2016).

5.S. Ali, A. Maciejewski, H. Siegel, K. Jong-Kook, Definition of a robustnessmetric for resource allocation, Parallel and Distributed Processing Symposium(2003) 22-26.

6.L. Canon, E. Jeannot, Evaluation and optimization of the robustness of DAGschedules in heterogeneous environments, IEEE Trans. Parallel Distrib. 21 (4)(2010) 532-546.

7.S. Srisakthi, A.P. Shanthi, Towards the design of a secure and fault tolerantcloud storage in a multi-Cloud environment, Inf. Secur. J. Global Perspect. 24(4-6) (2015) 109-117.

8.Top Threats to Cloud Computing v1.0. Cloud Security Alliance, 2010 (Online].Available:

https://cloudsecurityalliance.org/topthreats/csathreats.v1.0.pdf[access ed 29 June 2016)

9.O. A. Ben-Yehuda, M. Ben-Yehuda, A. Schuster, and D. Tsafrir, "Deconstructing amazon ec2 spot instance pricing," ACM Transaction Economy Computing, vol. 1, no. 3, pp. 16:1-16:20, Sept 2013.

10.D. Bernstein, E. Ludvigson, K. Sankar, S. Diamond, and M. Morrow, "Blueprint for the InterCloud - protocols and formats for cloud computing interoperability," in Proceedings of the 4th International Conference on Internet and Web Applications and Services, Venice, Italy, May 2009, pp. 328-336.

11.D. Bernstein, D. Vij, and S. Diamond, "An intercloud cloud computing economy - technology, governance, and market blueprints," in Proceedings of 2011 Annual SRII Global Conference (SRII), San Jose, California, USA, Apr. 2011, pp. 293 -299.

12.F. Black and M. Scholes, "The pricing of options and corporate liabilities," Journal of Political Economy, vol. 81, pp. 637-654, Jan 1973.

13.A. Bossenbroek, A. Tirado-Ramos, and P. M. A. Sloot, "Grid resource allocation by means of option 
contracts," Systems Journal, IEEE, vol. 3, no. 1, pp. 49-64, March 2009.

14.R. Buyya, R. Ranjan, and R. N. Calheiros, "InterCloud: Utility-oriented federation of cloud computing environments for scaling of application services," in Proceedings of the 10th International Conference on Algorithms and Architectures for Parallel Processing (ICA3PP'10), vol. 6081, Busan, South Korea, May 2010 , pp. 13-31.

15.R. Buyya, C. S. Yeo, S. Venugopal, J. Broberg, and I. Brandic, “Cloud computing and emerging IT platforms: Vision, hype, and reality for delivering computing as the 5th utility," Future Generation Computer Systems, vol. 25, no. 6, pp. 599-616, 2009

16.R. N. Calheiros, R. Ranjan, A. Beloglazov, C. A. F. D. Rose, and R. Buyya, "CloudSim: A toolkit for modeling and simulation of cloud computing environments and evaluation of resource provisioning algorithms," Software: Practice and Experience, vol. 41, no. 1, pp. 23-50, Jan. 2011.

17.R. N. Calheiros, A. N. Toosi, C. Vecchiola, and R. Buyya, "A coordinator for scaling elastic applications across multiple clouds," Future Generation Computer Systems, vol. 28, no. 8, pp. 1350-1362, Mar. 2012.

18.G. S. Campbell and J. M. Norman, Introduction to Environmental Biophysics. Springer Verlag, 1998.

19.A. Celesti, F. Tusa, M. Villari, and A. Puliafito, "How to enhance cloud architectures to enable cross-federation," in Proceedings of the 3rd International Conference on Cloud Computing (Cloud'10), Miami, USA, Jul. 2010, pp. 337-345.

20.S. Chaisiri, B.-S. Lee, and D. Niyato, "Optimal virtual machine placement across multiple cloud providers," in Proceedings of IEEE Asia-Pacific Services Computing Conference (APSCC'09). Jeju Island: IEEE, Dec. 2009, pp. 103-110.

21 